\title{
A Two-Parameter Recursion Formula For Scalar Field Theory
}

\author{
Y. Meurice and G. Ordaz \\ Department of Physics and Astronomy \\ University of Iowa, Iowa City, Iowa 52246, USA
}

\begin{abstract}
We present a two-parameter family of recursion formulas for scalar field theory. The first parameter is the dimension $(D)$. The second parameter $(\zeta)$ allows one to continuously extrapolate between Wilson's approximate recursion formula and the recursion formula of Dyson's hierarchical model. We show numerically that at fixed $D$, the critical exponent $\gamma$ depends continuously on $\zeta$. We suggest the use of the $\zeta$-independence as a guide to construct improved recursion formulas.
\end{abstract}


The renormalization group [1,2] method has shed light on the related questions of second order phase transitions and the infinite cut-off limit of field theories. The practical application of the method usually requires approximations. In the case of scalar field theory, a particularly simple approximate renormalization group transformation [1] is Wilson's "approximate recursion formula" (WARF in the following). The WARF is a simple integral equation with only one variable and has a free parameter which can be adjusted to approximate a $D$-dimensional theory. The WARF can be handled very easily using numerical methods or various perturbative expansions. The numerical value of the critical exponent $\gamma$ obtained [1] with the the WARF is 1.218 for $D=3$. The WARF can also be used to study non-perturbatively the cut-off dependence of the renormalized quantities. In the case $D=4$, this can be used to set triviality bounds [3] on the mass of a scalar particle.

The derivation of the WARF is a masterpiece of quantum calculation. Unfortunately, it is not based on an expansion in a small parameter and there no obvious way to restore order by order the details erased by the approximation. Clearly, one needs an organizing principle to improve the WARF. A group theoretical approach [⿴囗十 was proposed in the case of theories with quadratic interactions but this approach fails to control the proliferation of non-local terms in the case of quartic interactions. At the end of this letter, a new method of improvement will be suggested.

A renormalization group transformation "very closely connected" [5] to the WARF holds exactly for Dyson's hierarchical model [ [6] 8 . In the following, we use DHMRF as short for "Dyson's hierarchical model recursion formula". The DHMRF is also an integral equation with one variable, and it also has a free parameter expressible in terms of $D$. For definiteness, the WARF and DHMRF are given below by Eqs. (7-8) with $\zeta=1$ and $1 / D$ respectively. Roughly speaking [7], the WARF does in one step what the DHMRF does in $D$ steps. However, this is not an exact statement because [9] the value of $\gamma$ in $D=3$ is 1.300 . Consequently, the two models have different physical properties.

Before going further, we would like to emphasize that the difference between the two values of $\gamma$ quoted above is significantly larger than the errors involved in the numerical 
calculations. We have repeated Wilson's calculation with smaller integration steps, cutting the integral at larger values and using different criteria to determine criticality. We found that these changes affected $\gamma$ by less than 0.002 . We also used Wilson's numerical integration method for the DHRMF and found a value of 1.301 for $\gamma$. We confirmed [10] this result with errors smaller than 0.003 using the first 800 coefficients of the high-temperature expansion. Clearly, the difference between 1.22 and 1.30 is more than 25 times larger than the numerical errors involved in each calculation.

The WARF and the DHMRF can be seen as two approximate versions of the (much more complicated) renormalization group transformation for a scalar lattice model with nearest neighbor iterations. The WARF integrates $2^{D}$ field variables at a time keeping their sum constant while the DHMRF does the same thing but with only two field variables. The fact that different values of $\gamma$ are obtained with the two approximate methods sets a limit on the accuracy of the approximation. For comparison, the universal value of $\gamma$ in $D=3$ for nearest neighbor models is approximately 1.25 .

Ideally, one would like to construct a renormalization procedure for the nearest neighbor models corresponding to the integration of

$$
a \equiv 2^{\zeta D}
$$

field variables at a time and have all the physical quantities independent of $\zeta$. The quantity $2^{\zeta}$ is a scale factor which would play a role similar to the arbitrary scale parameter $\mu$ used in some version [11] of the Callan-Symanzik equations and where one obtains homogeneous differential equations of the form $\frac{d}{d \mu}$ (physical quantity) $=0$. In this letter, we make a first step in a similar direction, by introducing a recursion formula where $\zeta$ is arbitrary and which interpolates continuously between the $\operatorname{WARF}(\zeta=1)$ and the $\operatorname{DHMRF}(\zeta=1 / D)$.

This recursion formula can be constructed using Dyson's hierarchical model (DHM) as an explicit realization and then extending the results for arbitrary $\zeta$. In other words, this paragraph should first be read with $\zeta$ taking the fixed value $1 / D$ and considered as wellknown results written in a slightly unusual way. We call the sum of the fields in a cube 
containing $a^{l}$ sites $\phi_{l}$, with $l=1,2$.. corresponding to successive renormalization group transformations. We recall that $a$ is defined in Eq.(1) and takes the value 2 for DHM. At criticality, one has the scaling law 12

$$
<\left(\phi_{l}\right)^{2}>\propto(a b)^{2 l}
$$

where

$$
b \equiv 2^{-\frac{D-2}{2} \zeta}
$$

Calling $P_{l}(x) d x$ the probability for $\left(\phi_{l} /(a b)^{l}\right)$ to take a value between $x$ and $x+d x$, we see that at criticality,

$$
\rho_{l}=\int d x P_{l}(x) x^{2}
$$

tends to a constant for large $l$. On the other hand, in the high-temperature phase,

$$
<\left(\phi_{l}\right)^{2}>\propto(a)^{l}
$$

and $\rho_{l} \propto\left(a b^{2}\right)^{-l} . P_{l}(x)$ is the main object studied with the renormalization group method. Using the parametrization,

$$
P_{l}(x)=K e^{-(1 / a) Q_{l}(b x)},
$$

one can check that the recursion formula

$$
Q_{l+1}(x)=-a \ln \left[\frac{I_{l}(b x)}{I_{l}(0)}\right]
$$

with

$$
I_{l}(x)=\int_{-\infty}^{\infty} d y e^{-y^{2}-\frac{1}{2} Q_{l}(x+y)-\frac{1}{2} Q_{l}(x-y)}
$$

is equivalent to the DHMRF. The equivalence with the formulation usually found in the literature [8] is made clear by writing the recursion formula for the quantity $\psi_{l}(x)=I_{l-1}(b x)$. It is also clear that Eqs.(7-8) can be used for arbitrary value of $\zeta$. In the case, $\zeta=1$ 
one recovers immediately the WARF. In conclusion, Eqs. (7-8) can be used to interpolate continuously between the WARF and the DHMRF.

Note that $b$ in Eq.(3) can be seen as the scaling factor of a massless Gaussian field under a change of scale $2^{\zeta}$. This remark can be understood better by noting that the continuous (i.e. unregularized) version [13] of the massless Gaussian DHM is invariant under certain scale transformations. However, it is a discrete scale invariance. It seems plausible that discrete scale invariance allows log-periodic corrections [14] to the scaling laws. Such a corrections were indeed observed [10] very clearly in the case of DHM. Or goal is to find a formulation where the discrete scale invariance (and the unphysical features associated with it) would be replaced by a continuous scale invariance.

The critical behavior associated with the general recursion formula (7-8) can be studied with the usual methods. One starts with an initial function $Q_{0}(x)=r x^{2}+g x^{4}$ and for fixed $g$ determines the critical value $r_{c}$ by observing the transition in the behavior of $\rho_{l}$ defined above. Near this critical value, one obtains the linearized expression

$$
Q_{l}(x)=Q_{c}(x)+\left(r-r_{c}\right) \lambda^{l} R_{c}(x)
$$

where $\lambda$ is the largest eigenvalue of the renormalization group transformation. From this, one finds the critical exponent

$$
\gamma=\frac{\ln \left(a b^{2}\right)}{\ln (\lambda)}
$$

The numerical values for various values of $\zeta$ are displayed in Fig. 1. The results indicate that $\gamma$ is a continuous function of $\zeta$. As $\zeta$ becomes smaller, a larger number of iterations is necessary in order to obtain the critical value of $r$ with an acceptable accuracy. For practical purposes, the number of iterations is of the order of $15 / \zeta$. The limit $\zeta \rightarrow 0$ is thus difficult to reach computationally, however we have found no indication of a drastic change of behavior (e.g., a sudden drop to 1) in this limit.

We want to modify Eqs.(7-8) in order to get $\zeta$-independent physical quantities. In the following, we focus the discussion on the linear behavior given by Eq. (9). First, the 
$\zeta$-independence of $\gamma$ requires that $\lambda(\zeta)=(\lambda(1))^{\zeta}$. In addition, the renormalized coupling constants should also be $\zeta$-independent. Defining them with the procedure of Ref. [2] (which can be extended straightforwardly for arbitrary $a$ and $b$ ), we see that the functions $Q_{c}$ and $R_{c}$ should also be $\zeta$-independent. This requirement gives useful information concerning the corrections that need to be made to Eqs. (7-8). For instance if $Q_{c}$ is a $\zeta$-independent fixed point of such a corrected formula, one can set $\zeta=\zeta_{0}+\delta$ in the known part of the recursion formula. Expanding in $\delta$, one can construct, order by order in $\delta$, "counterterms" that cancel the $\zeta$-dependence. For instance at first order in $\delta$ we need to add corrections which have a form similar to the r.h.s of (8) but with insertions of $Q_{l}$ and its first derivative with easily calculable coefficients. The effects of these corrections will be investigated using numerical methods. We expect that this procedure can be used to systematically improve the WARF and applied to realistic calculations of the critical exponents and the triviality bounds. 


\section{REFERENCES}

[1] K. Wilson, Phys. Rev. B. 43185 (1971) ; K. Wilson and J. Kogut, Phys. Rep. 1275 (1974).

[2] K. Wilson, Phys. Rev. D 6, 419 (1972).

[3] P. Hazenfratz and J. Nager, Zeit. Phys. C 37, 477 (1988); T. E. Clarck, B. Heari and S. T. Love, Nucl. Phys. B402 628 (1993).

[4] Y. Meurice, Proceedings of the EPS Conference 1993, p. 89, J. Carr and M. Perrottet Eds. Editions Frontieres, 1994.

[5] K. Gawedzki and A. Kupianen, References of Lectures given at Les Houches Summer School 1984, K. Osterwalder and Stora Eds. North Holland, New York, 1986.

[6] F. Dyson, Comm. Math. Phys. 12, 91 (1969) ;

[7] G. Baker, Phys. Rev. B5, 2622 (1972).

[8] P. Bleher and Y. Sinai, Comm. Math. Phys. 45, 247 (1975) ; P. Collet and J. P. Eckmann, Comm. Math. Phys. 55, 67 (1977) and Lecture Notes in Physics 74 (1978) ; H. Koch and P. Wittwer, Comm. Math. Phys. 106495 (1986) , 138 (1991) 537 , 164 (1994) 627 .

[9] P. Collet, J.-P. Eckmann, and B. Hirsbrunner, Phys. Lett. 71B, 385 (1977).

[10] Y. Meurice, G. Ordaz and V. G. J. Rodgers, Phys. Rev. Lett. 75, 4555 (1995); Y. Meurice, S. Niermann and G. Ordaz, U. of Iowa preprint 96-10.

[11] D. Gross, Methods in Field Theory, section 4.4, R. Ballian and J. Zinn-Justin Eds., North Holland, Amsterdam, 1976 and references therein.

[12] G. Parisi, Statistical Field Theory (Addison Wesley, New-York, 1988).

[13] E. Lerner and M. Missarov, Theor. Math. Phys. 78 , 177 (1989). 
[14] H. Saleur, C.G. Sammis and D. Sornette, USC preprint 95-02. J. Feigenbaum and P. Freund, EFI preprint 95-58.

\section{Figure Captions}

Fig. 1: The critical exponent $\gamma$ as a function of $\zeta$ for $D=3$. 


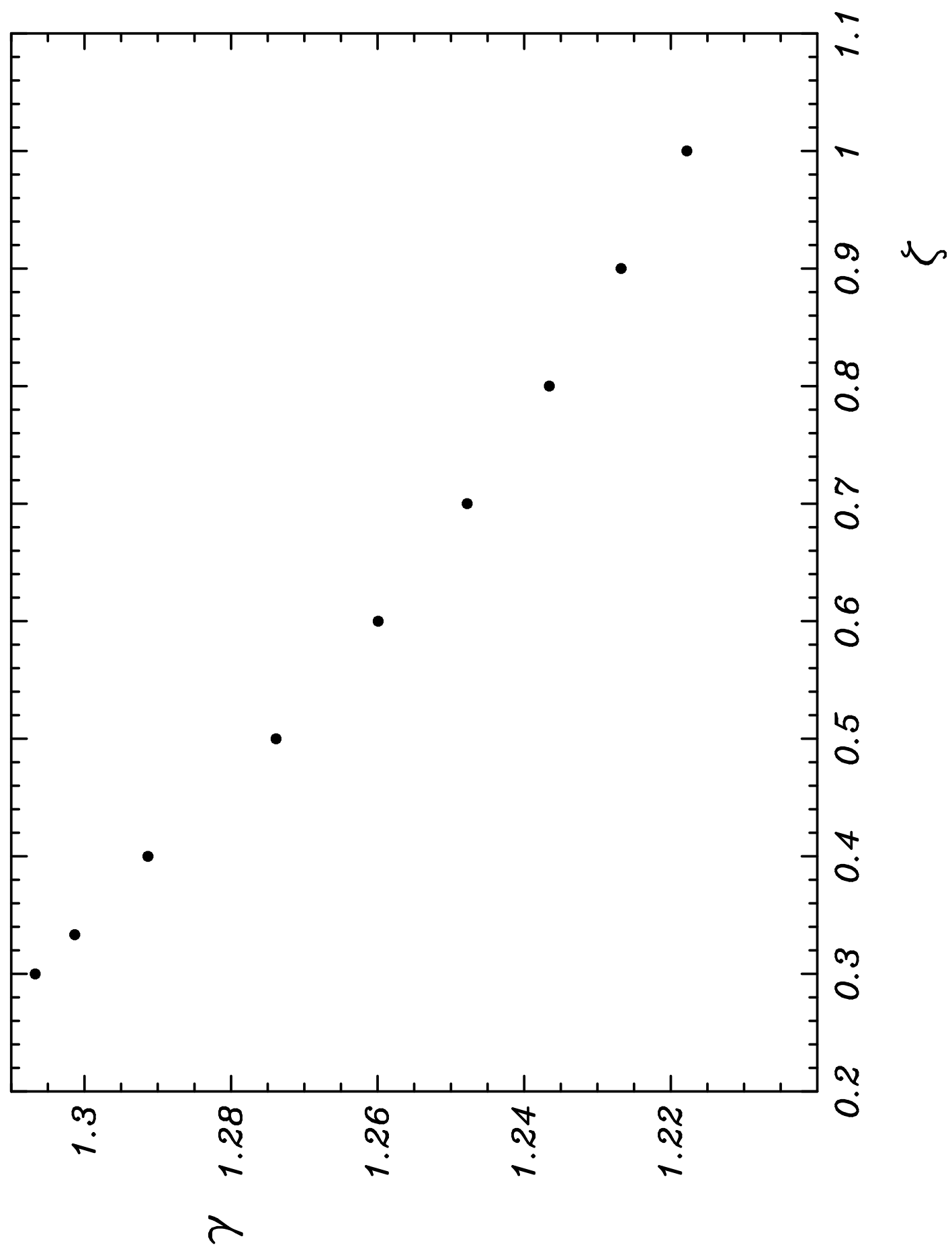

